\title{
Letter regarding Shen et al. entitled "The APE1 Asp148Glu polymorphism and colorectal cancer susceptibility: a meta-analysis"
}

\author{
Fei Liu $\cdot$ Yong-Gang Wei $•$ Wen-Tao Wang $\cdot$ Bo Li
}

Received: 22 October 2014 / Accepted: 3 November 2014 / Published online: 11 November 2014

(C) International Society of Oncology and BioMarkers (ISOBM) 2014

To the Editor,

We read with great interest the recent paper entitled "The APE1 Asp148Glu polymorphism and colorectal cancer susceptibility: a meta-analysis" published online in Tumor Biology by Shen et al. [1]. The investigators preformed a systematic review and meta-analysis to assess the effect of APE1 Asp148Glu polymorphism on the colorectal cancer susceptibility. Eight studies were included in their meta-analysis, and their study concluded that the APE1 Asp148Glu polymorphism was not associated with colorectal cancer susceptibility among Asians or Caucasians. It is an interesting and valuable article. Nevertheless, we have some comments related to this article.

To begin with, the investigators performed a systematical search only in English electronic databases (PubMed, Embase, Elsevier, and Web of Science databases). The small number of required papers would be an important limitation of this review. The investigators come from China; however, they did not perform a systematical search in Chinese electronic databases (such as CNKI, Weipu, Wanfang, and CBM databases). We recommend that more electronic databases should be included into this metaanalysis.

Furthermore, the data reported by Shen et al. for the study of Kasahara et al. [2] do not seem to be in line with the data provided by Kasahara et al. [2] in their original publication. The numbers reported by Shen et al. for TT and TG/GG in controls were 70 and 52; while the numbers in Kasahara et al's original publication were 70 and 51 . Also, the data reported by Shen et al. for the study of Berndt et al. [3] were not consistent

F. Liu $\cdot$ Y.-G. Wei $(\bowtie) \cdot$ W.-T. Wang $\cdot$ B. Li

Department of liver surgery, West China Hospital of Sichuan

University, 37 Guo Xue Road, Chengdu 610041, Sichuan Province,

China

e-mail: wyonggang688@163.com with the data provided by Berndt et al. in their original publication. The numbers reported by Shen et al. for TT, TG, GG in cases and controls were 175-364-153 and 204335-171; however, the numbers in their original publication for TT, TG, GG genotypes in cases and controls were 186387-166 and 222-357-178, respectively. After carefully studying, we found that Shen et al. only included the Caucasians population in that study. Why the other populations in that study were not included in the meta-analysis? We suggest that the investigators should include the total population in that study, which could avoid the potential bias in the metaanalysis.

In addition, the aim of this meta-analysis is to study the APE1 Asp148Glu polymorphism and colorectal cancer susceptibility. In the study identification and selection section, we found that the first inclusion criterion of this meta-analysis was "The articles evaluated the association between the APE1 Asp148Glu polymorphism and colorectal cancer susceptibility." However, Shen et al. included an article [3] which studied the APE1 Asp148Glu polymorphism and advanced colorectal adenoma risk. In our opinion, the advanced colorectal adenoma is not equal to colorectal cancer. According to the inclusion criteria of this meta-analysis, this article should be excluded.

Eventually, the authors concluded that the APE1 Asp148Glu polymorphism was not associated with colorectal cancer susceptibility among Asians or Caucasians. Because only three studies were from Asian population, there is a very high risk of reporting bias for the relationship of the APE1 Asp148Glu polymorphism and colorectal cancer susceptibility in Asian population. If the authors concluded that the APE1 Asp148Glu polymorphism might not be associated with colorectal cancer susceptibility among Asians, and additional studies based on Asian patients should be performed to reevaluate the association; the conclusion may be more convincing. 
In conclusion, to get a more comprehensive evaluation of APE1 Asp148Glu polymorphism and colorectal cancer susceptibility, more well-designed studies with larger sample sizes are needed.

Acknowledgments This work was supported by grant from the Application Foundation Project of Science and Technology Agency in Sichuan Province (No 2012JY0079).

\section{Conflicts of interest None}

\section{References}

1. Shen E, Liu C, Wei L, Hu J, Weng J, Yin Q, et al. The APE1 Asp148Glu polymorphism and colorectal cancer susceptibility: a meta-analysis. Tumour Biol. 2014;35:2529-35.

2. Kasahara M, Osawa K, Yoshida K, Miyaishi A, Osawa Y, Inoue N, et al. Association of MUTYH Gln324His and APEX1 Asp148Glu with colorectal cancer and smoking in a Japanese population. J Exp Clin Cancer Res. 2008;27:49.

3. Berndt SI, Huang WY, Fallin MD, Helzlsouer KJ, Platz EA, Weissfeld $\mathrm{JL}$, et al. Genetic variation in base excision repair genes and the prevalence of advanced colorectal adenoma. Cancer Res. 2007;67: 1395-404. 